\title{
Phosphorylation Site
}

National Cancer Institute

\section{Source}

National Cancer Institute. Phosphorylation Site. NCI Thesaurus. Code C14054.

Generally refers to amino acids residues that were covalently modified by phosphate group (PO4-). ( $\mathrm{NCl})$ 